\title{
EFFECTIVE ANT BASED ROUTING ALGORITHM FOR DATA REPLICATION IN MANETS
}

\author{
N.J. Nithya Nandhini ${ }^{1}$, S. Ramesh ${ }^{2}$ and P. Ganesh Kumar ${ }^{3}$ \\ ${ }^{1,2}$ Department of Computer Science and Engineering, Anna University - Regional Office, Madurai, India \\ E-mail: ${ }^{1}$ nithyajayakumaran@gmail.com, ${ }^{2}$ itz_ramesh87@yahoo.com \\ ${ }^{3}$ Department of Electronics and Communication Engineering, K. L. N College of Engineering, India \\ E-mail: ganesh_me@yahoo.com
}

\begin{abstract}
In mobile ad hoc network, the nodes often move and keep on change its topology. Data packets can be forwarded from one node to another on demand. To increase the data accessibility data are replicated at nodes and made as sharable to other nodes. Assuming that all mobile host cooperative to share their memory and allow forwarding the data packets. But in reality, all nodes do not share the resources for the benefits of others. These nodes may act selfishly to share memory and to forward the data packets. This paper focuses on selfishness of mobile nodes in replica allocation and routing protocol based on Ant colony algorithm to improve the efficiency. The Ant colony algorithm is used to reduce the overhead in the mobile network, so that it is more efficient to access the data than with other routing protocols. This result shows the efficiency of ant based routing algorithm in the replication allocation.
\end{abstract}

Keywords:

Selfish Node, Replica Allocation, Routing, Ant Colony Algorithm, Mobile Nodes, Selfish Node Detection

\section{INTRODUCTION}

MANET (Mobile Ad hoc Network) is a self-configuring system of mobile nodes, connected by wireless links. As the growth of mobile devices and their uses, has great attention to mobile ad hoc network. It is decentralized network with no fixed infrastructure [18]. The operation of MANETs does not rely on pre-existing infrastructure or base stations. The node in the mobile ad hoc network keeps on move randomly, changes the network topology [12]. Routing is the major problem in this type of dynamic environment. To improve the efficiency and to decrease the overhead, a novel approach is used based on the ant colony algorithm for data forwarding. The ant based approach is based on the swan intelligence. As the ant communication in real life, the routing is done in this mobile ad-hoc network. The communication among the ant is not direct in finding the path. So this approach is used in routing among the mobile nodes, where the nodes are not stable. In this paper, the discussion is about the mobile nodes behaviour in data forwarding and dealing with these types of mobile nodes. Nodes may act selfishly, to safe its battery power and its energy, those selfish nodes does not share its resources to the purpose of other nodes. In case, nodes share its resources for the benefits of other nodes, it may lose its power and become inefficient. So for this reason, some mobile nodes act selfishly. The nodes behaviour in the mobile ad hoc network may consider as three types [20]. They are as follows:

- Cooperative nodes

- Selfish nodes

- Partially selfish nodes

Type1 nodes - cooperative nodes: The type1 nodes are cooperative nodes. This may allow other nodes to use its resources. These nodes actively participate in the network. The nodes have to be cooperative in order to communicate. Cooperative nodes are active in route discovery and in data forwarding.

Type2 nodes-selfish nodes: Type 2 nodes are selfish nodes. These nodes do not share its resources for the benefits of other. But uses the resources of others nodes for their purpose. These selfish nodes may refuse to forward the data packets for other nodes to conserve their own energy.

Type3 nodes-partially selfish nodes: Type 3 nodes are complex to identify. These are not fully cooperative nodes or fully selfish node. Sometimes it may be cooperative and may be selfish too. Based on the battery power of the node, it may or may not act selfishly.

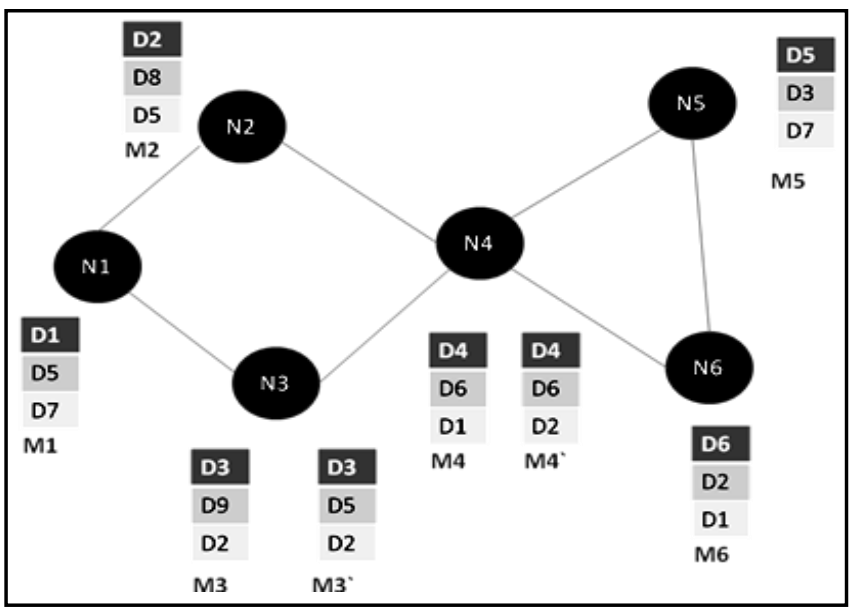

Fig.1. selfish replica allocations 


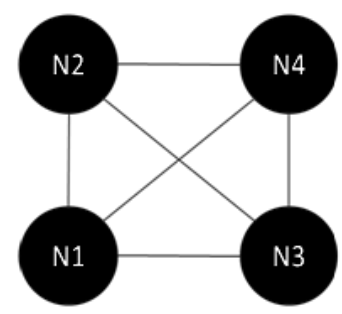

(a) Graph with non-selfish nodes

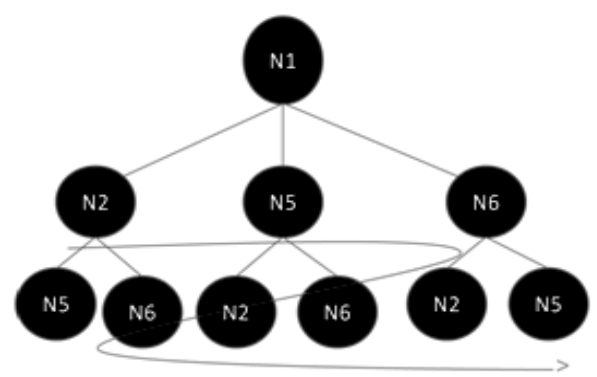

(b) SCF tree of N1 node

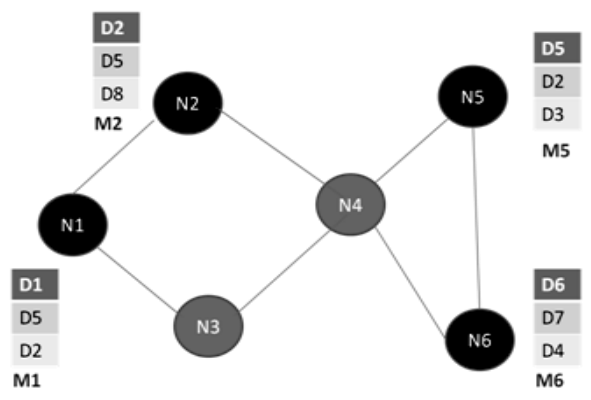

(c) Replica allocation with SCF tree

Fig.2. Replica allocation

Malicious vs. Selfish Nodes [19]: In mobile ad hoc network, there is no infrastructure. In order to maintain the communication mobile nodes have to cooperate. Selfish nodes cause non cooperation in network to save its power. These selfish nodes may not harm the network but it reduces the efficiency of the network.

Malicious nodes are not actually concerned with battery power to conserve energy. This malicious node will attack the network and these types of nodes are security threat to the network. Malicious nodes are active both in route discovery and launching attacks.

The remainder of this paper is organized as follows: Section 2 describes the selfish nodes in replica allocation and selfish node detection method. The replica allocation techniques are presented in Section 3. Section 4 discuss about routing algorithm based on ant colony algorithm (ACA) in data forwarding. The simulation result has been given in Section 5. Finally conclusion is given in section 6 .

\section{SELFISH NODES IN REPLICA ALLOCATION}

As the nodes in the mobile ad hoc network keeps on move randomly, changes the network topology and due to network partition some data may be inaccessible to some nodes [13]. This can be overcome by replicating the data item among the network rather than the original owner. When a data access to the data item thrives, then replica is created or when the mobile host connects to another mobile host which has the original or replica at relocation period. This replica allocated at nodes increases the data access and reduces the performance degradation. The average query delay or data item request may reduce due to data replication [10]. The non-cooperative nodes in the network, does not share its own memory space to allocate the replicas by other nodes.

The Fig.1 depicts [11] about the replica allocation to the mobile nodes. For instance, the mobile node N1 will be the owner data item D1 and also holds the replica D5 and D7, allocated by other nodes in the network. The non selfish nodes hold the replicas allocated by other node but the selfish nodes do not hold the replicas allocated by other nodes. For instance, the node N3 maintains the data set M3 instead of M3 and node N4 maintain the data set M4 instead of M4. This shows the selfish behaviour of node N3 and N4.

\subsection{DETECTING SELFISH NODES}

Selfish nodes can be detected based on the credit risk. Credit risk (CR) of each node is maintained by its neighbouring node depends on serving its query. The CR score can be estimated by the Eq.(1) [10],

Credit risk $=\frac{\text { Requested data not served by expected node }}{\text { Size of memory space } \& \text { No. of data item shared }}$

The modified equation can be given as [1],

$$
n C R_{i}^{k}=\frac{P_{i}^{k}}{\alpha * \frac{S S_{i}^{k}}{S_{i}}+(1-\alpha) * \frac{N D_{i}^{k}}{n_{i}}}
$$

where, $0 \leq \alpha \leq 1$.

Each node evaluates the CR score for other node, which they linked, by considering the selfish characteristics.

$n C R_{i}^{k}$ : Normalised credit risk of node $N_{k}$ by node $N_{i}$

$P_{i}^{k}$ : Ratio of selfish alarm of $N_{k}$ on node $N_{i}$

$S S_{i}^{k}$ : Size of $N_{k}$ 's shared memory space observed by $N_{i}$

$N D_{i}^{k}$ : Size of $N_{k}$ 's shared data items observed by $N_{i}$

$S_{i}$ : Size of memory space at node $N_{i}$

$n_{i}$ : Total number of data items accessed by $N_{i}$

$\alpha:$ System parameter

The threshold value is used to detect the level of $n C R_{i}^{k}$. If the normalised credit risk exceeds the threshold level then that node considered ad selfish node. To track the status of node, each parameter is updated at every query processing by other node.

\section{REPLICA ALLOCATION TECHNIQUES}

Replica allocation is done based on the self centred friendship tree (SCF). This SCF tree is encouraged by the human friendship management. As in real world, person can makes his/her own friendship on his/her discretion.

Self Centred Friendship tree (SCF) construction includes with the value of normalised credit risk score. Selfish nodes are eliminated and the SCF tree does not contain any selfish nodes. 
Thus each node has its own partial graph with only non selfish nodes as shown in Fig.2(a) [10].

For node $N$, it builds its own SCF tree on its own discretion as shown in Fig.2(b) [10]. The one hop connected nodes for node $\mathrm{N}$ are appended as child nodes. Then it iteratively checks child nodes of appended nodes.

Based on the SCF tree, replicas are allocated as shown in Fig.2(c). Node allocates replica at every relocation period. Replicas are allocated on the access frequency of group members. At first, a node determines the priority for allocating replicas. The priority is based on the Breadth First Search (BFS) order of SCF tree.

\section{ANT COLONY BASED ROUTING ALGORITHM}

The ant algorithm is based on the swarm intelligence. The swarm of ant that is used to find their communication path from the nest as a starting point, which is the source to the food place which is the destination [3]. The ant do not communicate directly, they use the pheromone to communicate between them. This communication is known as stigmergy.

Ant randomly moves to search the food. On its way it deposits the pheromone. With the pheromone concentration respective to time, the other ant follows the previous or most used path and finds the food. The pheromone concentration will be high in the path with shortest distance from the nest to food.

In mobile ad-hoc network, each node contains a routing table to maintain the routing information. The routing table has the structure as (initial node, last node, heuristics value) [1]. This heuristic value is used to select the route when their existing multipath. As in this routing, pheromone is the link place and to find the strength of the link with the existing information. The ants are the agents that simply used for communication in the network.

\subsection{BASIC ANT COLONY ALGORITHM}

Input: attractiveness, $\tau$, and visibility, $\eta$, for each edge;

\section{procedure $\mathrm{ACA}()$}

initialize the pheromone value;

while(not_termination)

generateSolutions()

localSearch ()

pheromoneUpdate()

end while

end procedure

Output: optimal path as solution.

The functionality ACA, finds the optimal path with the probability of pheromone.

The ant colony algorithm is more suitable to ad-hoc network than other routing algorithm. Because of the dynamic topology of network, some routing techniques are less efficient. This ant colony algorithm is based on the real food searching mechanisms of the ant [2]. The ant uses the pheromone deposits to find the path in real life. Likewise here artificial pheromone is used to find the shortest path in the network. The pheromone in the path is the indication of its usage in routing.

The ant colony algorithm based routing technique consists of three phases, such as

- Route Discovery Phase

- Route Maintenance Phase

- Route Failure Handling

\subsection{ROUTE DISCOVERY PHASE}

In route discovery phase, two agents are used, namely forward ant (FANT) and backward ant (BANT). Actually the route discovery phase is divided into two paths such as, forward path and the backward path. The ant agent used in forward routing that is from source to destination node to find the path is FANT. Likewise the BANT agent is used to find path from destination to source as in backward form [6].

The FANT agent establishes the pheromone track to the source node and the BANT agent establishes the pheromone track to the destination node. The FANT is created by the source node with a unique sequence number. The FANT is a small packet; any node can identify the duplicate node with the unique sequence number [5].

If a node receiving the FANT packet at the first time, it will make a record in its routing table. This record consists of parameter such as destination address, next hop, pheromone value.

The FANT is created by the source and send to the intermediate node from the source to destination node. The intermediate node gets the FANT and marks it in the routing table and sends it to the next hop.

When the FANT reaches the destination node, it is processed by extracting the FANT information by destination node and destroyed. Consequently it creates the BANT packet send it to source node. Once the sender receives the BANT successfully from the destination node, the path is generated and data can be forwarded in that path.

\subsection{ROUTE MAINTENANCE PHASE}

The second phase is the route maintenance phase; this phase is used to improve the route during the communication. It does not need any additional packet for maintenance phase. With the FANT and BANT packets, the path is maintained.

The initial pheromone value is changed in node with the usage of path for data forward. If the path is used by the FANT, then the pheromone value is increased. So the more usage, increase the pheromone value.

All the FANT packet has a unique sequence value, so there no duplication in FANT. If any duplication is received by the node then it can be identified and marked as DUPLICATE_ERROR and sends to the sender node back [7]. When the DUPLICATE_ERROR is received by the sender, it deactivates the link.

\subsection{ROUTE FAILURE HANDLING PHASE}

The route failure is done because of the mobility of nodes in the mobile ad-hoc network. The route failure is identified by the acknowledgement. If the acknowledgement is not received then 
it is considered as route failure and sends a ROUTE_ERROR message [4]. If the node gets the ROUTE_ERROR message, then it deactivates the link and set the pheromone value to 0 .

Then the node search for an alternative path to communicate using the information that has been recorded in the routing table. If there a possible another link is presented then the node sends the data through that link, otherwise it backtracks to the sender.

\section{SIMULATION RESULTS}

\subsection{SIMULATION ENVIRONMENT}

In this simulation experiments, the number of nodes is 40 . Each node will be the owner of a data and the data can be replicated among the other nodes in the network. The data forward is done on demand. The ant algorithm reduces the routing overhead that may increase the delivery rate during the data replication. The simulation result is compared with existing routing protocols such as DSR, AODV and DSDV in this environment.

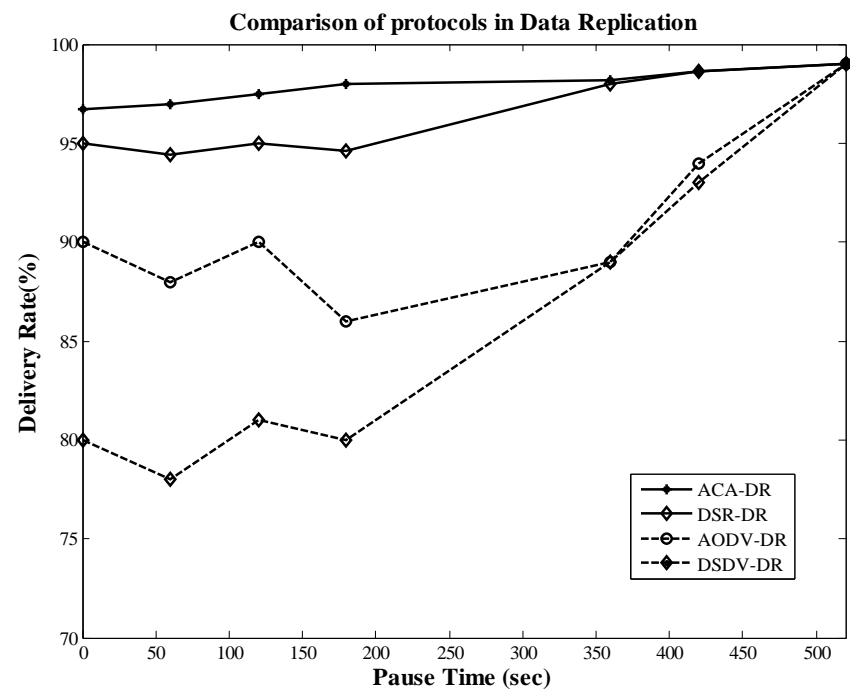

Fig.3. Comparison of routing protocols during the Data Replication (DR)

From the Fig.3, when the pause time is 0 , the nodes mobility will be high, therefore the delivery rate will be decreased in AODV-DR and DSDV-DR routing protocol. The delivery rate percentage using the DSR-DR routing protocol is closed to the ACA-DR protocol.

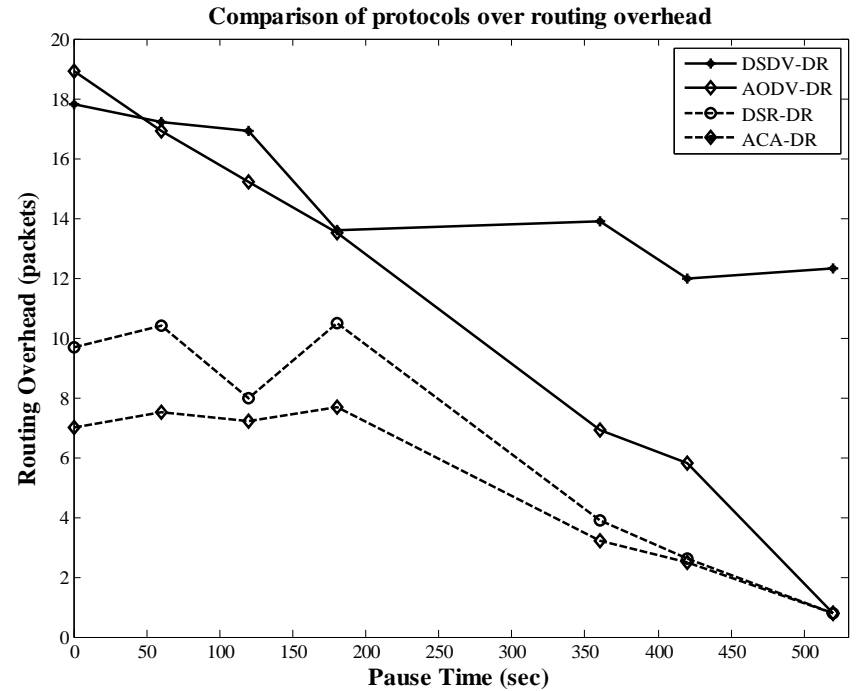

Fig.4. Comparison of protocols over routing overhead during Data Replication

The Fig. 4 shows the comparison of routing protocols. The ACA-DR has the less overhead in the mobile ad-hoc network than other existing routing protocols. Due to less overhead increase the delivery rate during the data replication.

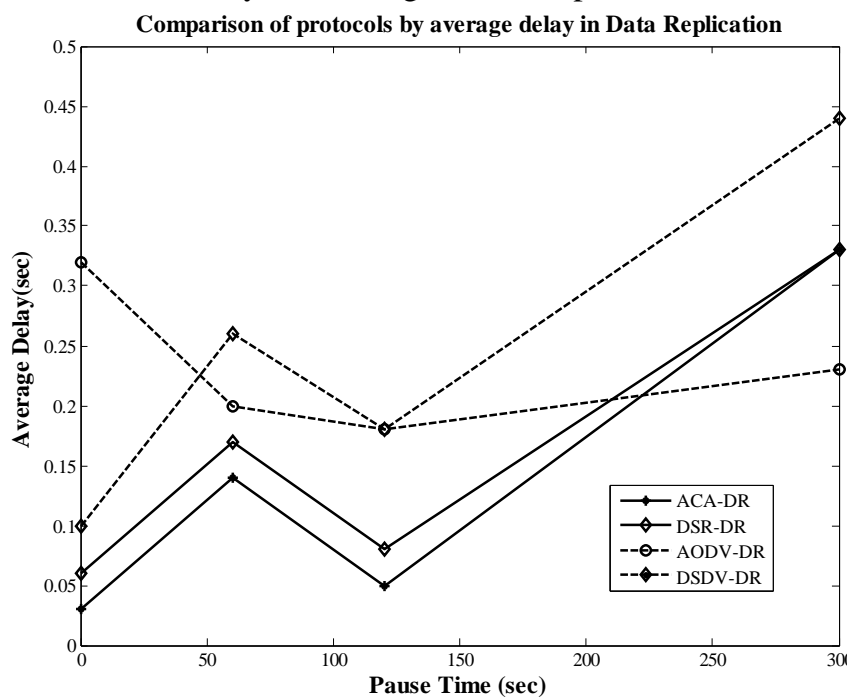

Fig.5. Comparison of protocols by average delay during Data Replication

The Fig.5 shows the comparison of routing protocols. The ACA-DR has the less average delay in the mobile ad-hoc network than other existing routing protocols.

\section{CONCLUSION AND FUTURE WORK}

Mobile ad-hoc network is more suitable where the installation is not feasible. This leads the complexity in routing due to the mobility of nodes in the network. To increase the data accessibility the data are replicated among nodes. Here the selfish problem in data allocation is addressed and the routing overhead is reduced. To reduce the routing overhead ant based approach, Ant Colony Algorithm (ACA) is used as the routing protocol. Due to less overhead, delivery rate is increased and 
thus improves the data allocation with respect to time. The simulation results shows that ACA based routing protocol performs better than existing protocols in the mobile ad-hoc network. The future work includes the scalability improvement in the data replication access.

\section{REFERENCES}

[1] Mesut Gunes, Udo Sorges and Imed Bouazizi, "ARA - The Ant-Colony Based Routing Algorithm for MANETs", Proceedings of International Conference Parallel Processing Workshops, pp. 79-85, 2002.

[2] Nada M. A. Al Salami, "Ant Colony Optimization Algorithm", UbiCC Journal, Vol. 4, No. 3, pp. 823-826, 2009.

[3] Zeng Yuan-Yuan and He Yan-Xiang, "Ant Routing Algorithm for Mobile Ad-hoc Networks Based on Adaptive Improvement", Proceedings of the International Conference on Wireless Communications, Networking and Mobile Computing, Vol. 2, pp. 678-681, 2005.

[4] Tiago Camilo, Carlos Carreto, Jorge Silva and Fernando Boavida, "An Energy-Efficient Ant-Based Routing Algorithm for Wireless Sensor Networks", Ant Colony Optimization and Swarm Intelligence, pp. 49-59, 2006.

[5] Ying Zhang, Lukas D. Kuhn and Markus P.J. Fromherz, "Improvements on Ant Routing for Sensor Networks", Ant Colony Optimization and Swarm Intelligence, pp. 154-165, 2004.

[6] Selcuk Okdem and Dervis Karaboga, "Routing in Wireless Sensor Networks Using an Ant Colony Optimization (ACO) Router Chip", Sensors, Vol. 9, No. 2, pp. 909-921, 2009.

[7] Zar Chi Su Su Hlaing and May Aye Khine, "An Ant Colony Optimization Algorithm for Solving Traveling Salesman Problem", Proceedings of International Conference on Information Communication and Management, Vol. 16, pp. 54-59, 2011.

[8] Ajay C Solai Jawahar," Ant Colony Optimization for Mobile Ad-hoc Networks", Parallel and Distributed Computing, Lecture 22, Rutgers University

[9] Bibhash Roy, Suman Banik, Parthi Dey, Sugata Sanyal and Nabendu Chaki, "Ant Colony based Routing for Mobile Ad-Hoc Networks towards Improved Quality of Services", Journal of Emerging Trends in Computing and Information Sciences, Vol. 3, No. 1, pp. 10-14, 2012.
[10] J. Choi, K. Shim, S. Lee and K. Wu "Handling Selfishness in Replica Allocation over a Mobile Ad Hoc Network", IEEE Transactions on Mobile Computing, Vol. 11, No. 2, pp. 278-291, 2012.

[11] T. Hara and S.K. Madria, "Data Replication for Improving Data Accessibility in Ad Hoc Networks", IEEE Transactions on Mobile Computing, Vol. 5, No. 11, pp. 1515-1532, 2006.

[12] T. Hara, "Effective Replica Allocation in Ad Hoc Networks for Improving Data Accessibility", Proceedings of $20^{\text {th }}$ Annual Joint Conference of the IEEE Computer and Communications Societies, Vol. 3, pp. 1568-1576, 2001.

[13] T. Hara and S.K. Madria, "Consistency Management Strategies for Data Replication in Mobile Ad Hoc Networks", IEEE Transactions on Mobile Computing, Vol. 8, No. 7, pp. 950-967, 2009.

[14] L. Anderegg and S. Eidenbenz, "Ad Hoc-VCG: A Truthful and Cost-Efficient Routing protocol for Mobile Ad Hoc Networks with Selfish Agents", Proceedings of the $9^{\text {th }}$ Annual International Conference on Mobile Computing and Networking, pp. 245-259, 2003.

[15] D. Hales, "From Selfish Nodes to Cooperative Networks Emergent Link-Based Incentives in Peer-to-Peer Networks", Proceedings of IEEE International Conference on Peer-to-Peer Computing, pp. 151-158, 2004.

[16] T.V.P. Sundarajan and A. Shanmugam, "Selfish Avoidance Routing Protocol for Mobile ad Hoc Network", International Journal of Wireless and Mobile Networks, Vol. 2, No. 2, pp. 74-86, 2010.

[17] M. Kargar and M. Ghodsi, "Truthful and Secure Routing in Ad Hoc Networks with Malicious and Selfish Nodes", International Journal of Security and its Applications, Vol. 3, No. 1, pp. 117-128, 2009.

[18] K. Dipali and K. Supriya, "Comparative Study of Techniques used for Detection of Selfish Nodes in Mobile Ad hoc Networks", International Journal of Soft Computing and Engineering, Vol. 1, No. 4, pp. 190-194, 2011.

[19] S. Buchegger and J.Y.L. Boudec, "Cooperative Routing in Mobile Ad-hoc Networks: Current Efforts against Malice and Selfishness", in: Lecture Notes on Informatics, Mobile Internet Workshop, 2002.

[20] Pietro Michiardi and Refik Molva, "Simulation-based Analysis of Security Exposures in Mobile Ad Hoc Networks", Proceedings of European Wireless Conference, 2002. 